\title{
Acetylshikonin Inhibits Colorectal Cancer Growth via PI3K/Akt/mTOR Signaling Pathway
}

\author{
Yuzhen Zhu1", Yu Zhong2*, Yu Zhou ${ }^{3}$, Yanyan Liu', Qionglin Huang², Zhe Huang5, \\ Yongcun Wang6, Hua Ye1, Xiaobing Zeng',7\#, Xuebao Zheng ${ }^{1,8 \#}$
}

\author{
${ }^{1}$ Guangdong Key Laboratory for Research and Development of Natural Drugs, Guangdong Medical University, \\ Zhanjiang, China \\ ${ }^{2}$ Analysis Center of Guangdong Medical University, Zhanjiang, China \\ ${ }^{3}$ Department of Gastroenterology, Affiliated Hospital of Guangdong Medical University, Zhanjiang, China \\ ${ }^{4}$ Department of Clinical Laboratory, Affiliated Run Shaw Hospital of Zhejiang University School of Medicine, \\ Hangzhou, China \\ ${ }^{5}$ Department of Gastrointestinal Surgery, Affiliated Hospital of Guangdong Medical University, \\ Zhanjiang, China \\ ${ }^{6}$ Oncology Center, Affiliated Hospital of Guangdong Medical University, Zhanjiang, China \\ ${ }^{7}$ Center Lab of Longhua Branch, Shenzhen People's Hospital, 2nd Clinical Medical College of Jinan University, \\ Shenzhen, China \\ ${ }^{8}$ Mathematical Engineering Academy of Chinese Medicine, Guangzhou University of Chinese Medicine, \\ Guangzhou, China \\ Email: "xuebaozhenggzy@126.com, "zengxiaobin1983@163.com
}

How to cite this paper: Zhu, Y.Z., Zhong, Y., Zhou, Y., Liu, Y.Y., Huang, Q.L., Huang, Z., Wang, Y.C., Ye, H., Zeng, X.B. and Zheng, X.B. (2018) Acetylshikonin Inhibits Colorectal Cancer Growth via PI3K/Akt/mTOR Signaling Pathway. Chinese Medicine, 9, 126-143.

https://doi.org/10.4236/cm.2018.93008

Received: June 19, 2018

Accepted: August 5, 2018

Published: August 8, 2018

Copyright (c) 2018 by authors and Scientific Research Publishing Inc. This work is licensed under the Creative Commons Attribution International License (CC BY 4.0).

http://creativecommons.org/licenses/by/4.0/

c) (i) Open Access

\begin{abstract}
Background: Acetylshikonin, a major constituent isolated from Arnebia euchroma, is a potential candidate for anti-colorectal cancer drugs. However, the potential activity and underlying mechanism of Acetylshikonin against colorectal cancer remain unclear. Methods: In this study, Acetylshikonin was isolated from the active $\mathrm{CHCl} 3$ extract of Arnebia euchroma using activity-guided screening, and elucidated by the extensive spectroscopic analysis and comparison with literature data. Human colorectal cancer cells HT29, DLD-1, HCT116 or Caco-2 were exposed to different concentrations of Acetylshikonin (6.25 - 100 $\mu \mathrm{g} / \mathrm{mL}$ ) for 24 or $48 \mathrm{~h}$. Cell viability, cell apoptosis and cell cycle distribution were detected. The activity of Acetylshikonin and potential mechanism of the phosphoinositide 3-kinase (PI3K)/Akt/mammalian target of rapamycin (mTOR) pathway were evaluated in vitro and vivo. Results: We found that Acetylshikonin exhibited remarkable anti-proliferative activity in a dose-dependent manner against HT29 cells with the IC50 values of 60.82 $\mu \mathrm{g} / \mathrm{ml}$ and $30.78 \mu \mathrm{g} / \mathrm{ml}$ at $24,48 \mathrm{~h}$, respectively. Moreover, Acetylshikonin induced cell cycle arrest at G0/G1 phase and early apoptosis through inhibition of $\mathrm{PI} 3 \mathrm{~K} / \mathrm{Akt} / \mathrm{mTOR}$ pathway. Furthermore, the assays of cell inhibition, early
\end{abstract}

*Yuzhen Zhu and Yu Zhong contributed equally to this work. 
apoptosis and G0/G1 phase distribution showed that suppression of the PI3K/Akt pathway using LY294002 enhanced the anti-cancer effect of Acetylshikonin. Similarly, Acetylshikonin also decreased the growth of tumour in colorectal cancer xenografts in mice through PI3K/Akt/mTOR pathway. Conclusions: To sum up, these new findings provided a framework for further exploration of Acetylshikonin which possessed the potential antitumor activity by inhibiting PI3K/Akt/mTOR pathway.

\section{Keywords}

Arnebia euchroma, Acetylshikonin, Colorectal Cancer, Apoptosis, PI3K/Akt/mTOR Pathway

\section{Background}

Colorectal cancer (CRC) is one of the most common malignancies in the world, accounting for approximately 1.36 million new cases worldwide every year [1]. Although advances in detection and local control with surgery, radiation and chemotherapy, the overall survival rate of colorectal cancer patients has not improved significantly during the past several decades. Chemotherapy is a common therapeutic strategy after surgery, and the marketed chemotherapy drugs kill tumour cells through competitive inhibition of nucleotide synthesis or cytotoxicity. However, these drugs often cause vomiting, myelosuppression, drug resistance and other adverse effects [2]. Therefore, searching for novel anticancer components of traditional Chinese medicine has become a very interesting option for developing anti-colorectal cancer drugs.

Shikonin, a natural occurring naphthoquinone, has potent anti-tumour activity. Shikonin is able to inhibit cell proliferation and induces apoptosis in several human malignancies such as gastric cancer [3], non-small cell lung cancer [4], pancreatic cancer [5] and so on. However, certain limitations exist, including no clear target and liver and kidney toxicity [6]. Therefore, highly anti-cancer and low or even nontoxic shikonin-like compounds cause our concerns. Shikonin derivatives have exhibited antivirus, antioxidation, anti-inflammatory, anti-fertility, and other pharmacological effects in previous studies [7]. Additionally, shikonin derivatives have strong anti-tumour activities with few adverse effects, which make shikonin derivatives as promising anti-tumour agents [8].

In this study, Acetylshikonin as a major chemical component was extracted and isolated from the dried roots of Arnebia euchroma by preparing HPLC. Acetylshikonin, as one of shikonin derivatives, has been shown to possess anticancer activity [9] [10] with less toxicity [6]. However, the potential activity and underlying mechanism of Acetylshikonin in colorectal cancer inhibition remain unknown. In our study, we examined the anticancer activity of Acetylshikonin in human colorectal cancer cells in vitro and in human colorectal cancer xenografts NOD/SCID mice in vivo. These new results provided a 
framework for further exploration of Acetylshikonin which possessed the potential antitumor activity by inhibiting PI3K/Akt/mTOR pathway.

\section{Methods}

\subsection{General}

Optical rotations were measured using a JASCO P-1030 (Tokyo, Japan) automatic digital polarimeter. NMR spectra were recorded on a Bruker DPX-400 spectrometer $\left(400 \mathrm{MHz}\right.$ for ${ }^{1} \mathrm{H}$ NMR and $100 \mathrm{MHz}$ for ${ }^{13} \mathrm{C}$ NMR, Karlsruhe, Germany) using standard Bruker pulse programs. Chemical shifts were showed as the $\delta$-value with reference to tetramethylsilane (TMS) as an internal standard. Electrospray ionization mass spectrometry (ESI-MS) data were obtained on a Bruker Esquire LC 200-Ion trap mass spectrometer (Bruker, Karlsruhe, Germany). And Sephadex LH-20 (Pharmacia, Sweden), silica gel (Qingdao Ocean Chemical Co., Ltd., Qingdao, China), and octadecylsilane (ODS) silica gel (Macherey-Nagel, Duren, Germany) were used for column chromatography. HPLC was performed using an ODS silica gel column (Cosmosil 5C18-MS-II, $20 \times 250$ $\mathrm{mm}$, Nacalai Tesque). All other chemicals were analytical or HPLC grade and obtained from Shanghai Chemical Reagents Co., Ltd. (Shanghai, China). Human colon cancer cell lines HT29, DLD-1, HCT116 and Caco-2 were purchased from the ATCC (PA, USA). These cells were grown in RPMI 1640 (Invitrogen, CA, USA) supplemented with $10 \%(\mathrm{v} / \mathrm{v})$ fetal bovine serum (Invitrogen, CA, USA) and $1 \%$ penicillin-streptomycin (Invitrogen, CA, USA). MTT powder was obtained from Sigma (Aldrich, USA). The Cell Counting Kit-8 assay was purchased from Dojindo Laboratories (Kumamoto, Japan). A Cycle Test Plus DNA reagent kit and Annexin V-FITC Apoptosis Detection Kit were purchased from BD Biosciences Pharmingen (NJ, USA). Total RNA extraction kit was procured from Omega (R6834-02, GA, USA). The qRT-PCR was performed using EASY spin Plus Total RNA Extraction Kit (Aidlab, Beijing, China), Transcriptor First Strand cDNA Synthesis Kit (Roche, Basel, Switzerland) and SYBR Premix ExTaq kit (Takara, Dalian, China). Antibodies against PI3K, p-PI3K, Akt, p-Akt, mTOR, $\beta$-Actin were purchased from Cell Signalling Technology (MA, USA). BCA Protein Assay Kit, RIPA cell lysates, PMSF and Hematoxylin and Eosin Staining Kit were obtained from Beyotime (Shanghai, China). Irinotecan was obtained from Qilu Pharmaceutical Co. Ltd (Hainan, China).

\subsection{Extraction and Isolation}

The roots of A. euchroma were purchased in Haozhou, Anhui Province, China, and subjected to taxonomic identification with Voucher specimens (No. 161001) deposited at Center Lab of Longhua Branch, Shenzhen People's Hospital, 2nd Clinical Medical College of Jinan University in Shenzhen, China. The roots of $A$. euchroma (7.5 kg) were extracted three times with $95 \%$ ethanol. The solvent was removed under vacuum to yield the crude extract $(1150 \mathrm{~g})$. The crude extract was resuspended in water and partitioned with chloroform $(3 \mathrm{~L} \times 3)$, ethyl ace- 
tate $(3 \mathrm{~L} \times 3)$ and water-saturated $n$-butanol $(3 \mathrm{~L} \times 3)$ gradually to afford 178.21 $\mathrm{g}, 62.01 \mathrm{~g}$ and $254.35 \mathrm{~g}$ of dried organic extracts, respectively.

Bioactivity-guided fractionation was used for the isolation work. On the basis of the bioactive results of the extracts, the chloroform fraction with the most potential activity was fractionated over a silica gel (200 - 300 mesh) column eluting with a gradually amount of $\mathrm{MeOH}$ in $\mathrm{CHCl}_{3}$ to give 5 fractions. The $\mathrm{CHCl}_{3}-\mathrm{MeOH}$ (40: 1$)$ elution (25.0 g) was further purified by a Sephadex LH-20 column $(6 \times 88$ $\mathrm{cm}, 680 \mathrm{~g}$; Solvent system: $\left.\mathrm{CHCl}_{3}-\mathrm{MeOH}, 7: 3\right)$ to obtain three major fractions, 1a-1c. Fraction 1a (15.0 g) was applied to a preparative HPLC with $89 \%$ methanol (containing $0.1 \% \mathrm{CF}_{3} \mathrm{COOH}, \mathrm{pH} 3.0$ ) to give fractions 1a1-1a3, and Acetylshikonin $(1122.15 \mathrm{mg})$ were purified by preparative $\mathrm{HPLC}(83 \% \mathrm{MeOH}$ and $83 \% \mathrm{MeOH}$, containing $0.1 \% \mathrm{CF}_{3} \mathrm{COOH}, \mathrm{pH} 3.0$ ) at $8 \mathrm{ml} / \mathrm{min}$ from Fraction $1 \mathrm{a} 2$ (1421.1 mg).

\subsection{Acetylshikonin}

Acetylshikonin was a red amorphous powder; ESI-MS m/z $331[\mathrm{M}-\mathrm{H}]^{-} ;[\alpha]_{25}^{D}$ $+151.2\left(\mathrm{c} 0.20, \mathrm{CHCl}_{3}\right) ;{ }^{1} \mathrm{H} \mathrm{NMR}\left(\mathrm{CDCl}_{3}, 400 \mathrm{MHz}\right)$ data: $6.99(1 \mathrm{H}, \mathrm{s}, \mathrm{H}-3), 7.18$ (2H, s, H-6, 7), $12.42(1 \mathrm{H}, \mathrm{s}, 6-\mathrm{OH}), 12.58(1 \mathrm{H}, \mathrm{s}, 5-\mathrm{OH}), 6.03(1 \mathrm{H}, \mathrm{dd}, \mathrm{J}=7.2$, $\left.4.4 \mathrm{~Hz}, \mathrm{H}-1^{\prime}\right), 2.46\left(1 \mathrm{H}, \mathrm{m}, \mathrm{H}-2^{\prime}\right), 2.62\left(1 \mathrm{H}, \mathrm{m}, \mathrm{H}-2^{\prime}\right), 5.12(1 \mathrm{H}, \mathrm{t}, \mathrm{J}=7.6 \mathrm{~Hz}$, H-3'), 1.69 (3H, s, 5'- $\left.\mathrm{CH}_{3}\right), 1.58\left(3 \mathrm{H}, \mathrm{s}, 6^{\prime}-\mathrm{CH}_{3}\right), 2.14\left(3 \mathrm{H}, \mathrm{s}, \mathrm{H}-2^{\prime \prime}\right) ;{ }^{13} \mathrm{C} \mathrm{NMR}$ $\left(\mathrm{CDCl}_{3}, 100 \mathrm{MHz}\right)$ data: 178.1 (C-1), 148.2 (C-2), 136.1 (C-3), 176.6 (C-4), 111.6 (C-5), 169.7 (C-6), 131.5 (C-7), 132.7 (C-8), 167.0 (C-9), 111.8 (C-10), 69.5

(C-1'), 32.8 (C-2'), 117.6 (C-3'), 132.9 (C-4'), 17.9 (C-5'), 25.7 (C-6'), 167.6 $(\mathrm{C}-1$ "), 20.9 (C-2").

\subsection{Cell Culture}

Four human colon carcinoma cell lines including HT29, DLD-1, HCT116, Caco-2 were cultured in the Dulbecco's modified Eagle medium supplemented with $10 \%$ fetal bovine serum, $100 \mu \mathrm{g} / \mathrm{ml}$ penicillin, and $100 \mu \mathrm{g} / \mathrm{mL}$ streptomycin. The cells were incubated at $37^{\circ} \mathrm{C}$ in a humidified atmosphere with $5 \% \mathrm{CO}_{2}$.

\subsection{Methyl Thiazolyl Tetrazolium (MTT) Assay}

HT29 cells $\left(5 \times 10^{3}\right.$ cells/well $)$ were seeded in 96-well plates and cultured for 24 h. Cells were treated with various concentrations of Acetylshikonin $(6.25,12.5$, $25,50,100 \mu \mathrm{g} / \mathrm{mL})$ for 24 or $48 \mathrm{~h} .20 \mu \mathrm{l} \mathrm{MTT}$ solution $(0.5 \mathrm{mg} / \mathrm{mL}$ in PBS) was supplemented into each well and incubated for an additional 4 hours. The supernatant was then discarded, and $100 \mu \mathrm{L}$ DMSO was added to dissolve the formazan crystals. Optical density was read at a wavelength of $490 \mathrm{~nm}$ on a microplate reader. Cytotoxicity of Acetylshikonin was assessed as described previously, and calculated by the following formula:

$$
\text { Inhibitory rate }(\%)=\left(O D_{\text {Control }}-O D_{\text {Sample }}\right) /\left(O D_{\text {Control }}-O D_{\text {Blank }}\right) \times 100 \%
$$

\subsection{Cell Proliferation Assay}

Cell proliferation analysis was conducted using the Cell Counting Kit-8 (CCK-8) 
according to the manufacturer's protocol. Each cell line including Caco-2, HCT116, DLD-1, HT29 was seeded into 96 -well plates $\left(5 \times 10^{3}\right.$ cells/well). After $24 \mathrm{~h}$, the medium was replaced with fresh medium and then adhered cells were treated with different concentrations of Acetylshikonin $(6.25,12.5,25,50,100$ $\mu \mathrm{g} / \mathrm{mL}$ ) for $48 \mathrm{~h}$. The CCK- 8 solution was added to each well and incubated for additional $2 \mathrm{~h}$. The number of viable cells was quantified by assessing the absorbance $(450 \mathrm{~nm})$ using Multiskan Spectrum Microplate Reader. The inhibitory rate of cell proliferation was calculated by the following formula:

Inhibitory rate $(\%)=1-\left(O D_{\text {Sample }}-O D_{\text {Blank }}\right) /\left(O D_{\text {control }}-O D_{\text {Blank }}\right) \times 100 \%$

\subsection{Apoptosis Detection Assay}

HT29 (5 × $10^{5}$ cells/well) in 6-well culture plates were exposed to various concentrations of Acetylshikonin for 24 or $48 \mathrm{~h}$. After that, we harvested the cells and supernatants, and washed them in PBS and resuspended $1 \times 10^{6}$ cells $/ \mathrm{mL}$ in $1 \times$ Binding Buffer. $5 \mu \mathrm{L}$ FITC-conjugated Annexin V and $5 \mu$ Propidium lodide (PI) were added to the $100 \mu \mathrm{L}$ cell suspension. After incubation for 15 minutes at room temperature in the dark, cells were then analyzed by flow cytometer. The amount of early apoptosis, late apoptosis, and necrosis was determined as the percentage of Annexin $\mathrm{V}^{+} / \mathrm{PI}^{-}$, Annexin $\mathrm{V}^{+} / \mathrm{PI}^{+}$, and Annexin $\mathrm{V}^{-} / \mathrm{PI}^{+}$cells, respectively. Each experiment was performed in triplicate.

\subsection{Cell Cycle Analysis Assay}

HT29 cells were trypsinized with $0.25 \%$ Trypsin EDTA at room temperature after pretreatment with various concentration of Acetylshikonin for $24 \mathrm{~h}$, and washed three times in buffer solution. Cell cycle distribution was detected by Cycletest Plus DNA Reagent kit. The percentages of cells in the different cell cycle phases (G0/G1, S, G2/M) were calculated using Flow cytometry.

\subsection{Real-Time Polymerase Chain Reaction (RT-PCR)}

HT29 cells were treated with Acetylshikonin at different concentration of 0 , $25,50,100 \mu \mathrm{g} / \mathrm{ml}$ for $48 \mathrm{~h}$. Total RNA was extracted by Omega total RNA extraction kit and the total RNA was reverse transcribed using TaKaRa reverse transcription kit. Using reverse transcribed $\mathrm{cDNA}$ as the template and $\beta$-actin as the endogenous control. The amplification reactions were carried out according to the one-step SYBR1 prime Script1 RT-PCR II kit instructions. Amplification was performed with an ABI 7500 real-time PCR thermocycler. Real-time PCR reaction system: $2 \mu \mathrm{l}$ cDNA product, $0.4 \mu \mathrm{l}$ each of forward and reverse primers, $10 \mu \mathrm{l}$ Taq Master Mix, $6.8 \mu \mathrm{l}$ RNase-free water, $0.4 \mu \mathrm{l}$ ROX Reference Dye $(50 \times)$, total volume $20 \mu \mathrm{l}$. The primer sequences that were used are shown in Table 1 . Reaction conditions: pre-denaturation at $95^{\circ} \mathrm{C}$ for $30 \mathrm{~s}$, $95^{\circ} \mathrm{C}$ for $5 \mathrm{~s}, 57^{\circ} \mathrm{C}$ for $34 \mathrm{~s}$ and repeat for 35 cycles. Each sample repeated 3 times. The relative expression of each gene was calculated using the $2^{-\Delta \Delta \mathrm{Ct}}$ method. 
Table 1. Premer sequences of the genes.

\begin{tabular}{cl}
\hline Gene names & \multicolumn{1}{c}{ Premer $\left(5^{\prime}-3^{\prime}\right)$} \\
\hline \multirow{2}{*}{ Akt } & Forward: CTTGCTTTCAGGGCTGCTCA \\
& Reverse: TACACGTGCTGCCACACGATAC \\
& Forward: CTGTCAATCGGTGACTGTGTGG \\
PI3K & Reverse: AAACAGGTCAATGGCTGCATCATA \\
& Forward: AGAAACTGCACGTCAGCACCA \\
mTOR & Reverse: CCATTCCAGCCAGTCATC TTTG \\
& Forward: GCTTCTAGGCGGACTGTTAC \\
& Reverse: CCATGCCAATGTTGTCTCTT
\end{tabular}

\subsection{Western Blot Analysis}

HT29 $\left(5 \times 10^{5}\right.$ cells/well $)$ in 6 -well culture plates were exposed to treat with Acetylshikonin for $48 \mathrm{~h}$. Both adherent and floating cells were collected. The tumour tissue protein was purified according to the reported method [11]. Western blotting used standard protocols. Proteins were separated by SDS-PAGE and transferred onto nitrocellulose membranes that were blocked with 5\% non-fat milk in TBST, and incubated with primary antibodies: Akt, p-Akt, PI3K, p-PI3K, mTOR, $\beta$-actin (Cell Signaling Technology, MA, USA). Then we add HRP-conjugated polymer-tagged secondary antibodies (Abcam, MA, USA). Lastly, the protein bands were captured using Western blotting detection system (DNR Bio-Imaging Systems, Jerusalem, Israel). Signal intensity was quantified by densitometry with the Gel-pro Analyzer (Media Cybernetics, Rockville, MD, USA). All experiments were done in triplicate.

\subsection{Inhibition of PI3K/Akt Pathway}

HT29 cells were pretreated with LY294002 (10 mM, Selleck Chemicals, TX, USA), for $30 \mathrm{~min}$, then co-treated with $50 \mu \mathrm{g} / \mathrm{mL}$ Acetylshikonin for further 48 h. Then cell viability, cell apoptosis, cell cycle arrest and expressions of PI3K/Akt proteins were measured in terms of the above experimental methods.

\subsection{Tumour Xenograft in NOD/SCID Mice}

A total of 16 male NOD/SCID mice with body weights of $10-12 \mathrm{~g}$, were purchased from model animal research center of Nanjing University and maintained in a specific pathogen-free environment. Animal experiments were approved by the Guangdong Medical University Institutional Animal Care and Use Committee. After 2 weeks, mice were injected with DLD-1 cells $\left(1 \times 10^{7}\right.$ cells in $0.2 \mathrm{~mL}$ volume) into the subcutaneous tissue of the back region. Tumour volume was measured by calliper every two day and calculated by the formula: (length $\times$ width $\times$ width)/2. Mice began to treat with drugs when tumours reached an average size of $62.5 \mathrm{~mm}^{3}$. The tumour-bearing mice were divided randomly into the following three groups and given different drugs by intraperitoneal injection for 13 days: control (1\% DMSO, every two days), Acetylshiko- 
nin $(20 \mathrm{mg} / \mathrm{kg}$ in $1 \%$ DMSO, every two days), irinotecan $(66.7 \mathrm{mg} / \mathrm{kg}$, every four days). All mice were killed on Day 13, and the tumours were segregated, measured, weighed, and stored in liquid nitrogen for later use.

\subsection{Hematoxylin/Eosin (H \& E) Staining}

Tumours were collected, fixed in formalin and embedded in paraffin. Tissue sections (5 $\mu \mathrm{m}$ in thickness) were prepared according to standard protocols for H\&E staining. After staining, the tissue slices were viewed under microscopy with the Olympus DP controller software program (Tokyo, Japan).

\subsection{Statistical Analysis}

All experiments were performed at least three times, independently. The results were analysed using GraphPad Prism version 6.0 to perform one-way ANOVA. $P$ value less than 0.05 was considered statistically significant.

\section{Results and Discussion}

\subsection{Isolation and Identification of Acetylshikonin}

Acetylshikonin (Figure 1) was isolated from the extracts of A. euchroma by bioassay-guided fractionation, and identified using spectral analysis by ${ }^{1} \mathrm{H}$ and ${ }^{13} \mathrm{C}$ NMR and comparison with literature data [12].

\subsection{Acetylshikonin Exhibited Anti-Proliferative Activity against Human Colorectal Cancer Cells}

To explore the effects of Acetylshikonin on colorectal cancer cells proliferation, HT29 cells were exposed to different concentration of Acetylshikonin for 24 or $48 \mathrm{~h}$, and cell viability was measured using the MTT assay. As shown in Figure 2(a) and Figure 2(b), Acetylshikonin showed significant inhibition against the HT29 cell with $\mathrm{IC}_{50}$ values of 60.82 and $30.78 \mu \mathrm{g} / \mathrm{ml}$ at 24 and $48 \mathrm{~h}$, respectively. Also, human colorectal cancer cells including Caco-2, HCT116, DLD-1 and HT29 were treated with Acetylshikonin for $48 \mathrm{~h}$, and cell proliferation analysis was conducted by Cell Counting Kit-8. It was found that Acetylshikonin led to a dose-dependent inhibition of cell proliferation in all four cancer cell lines. Taken together, these results suggest that Acetylshikonin inhibits the growth of colorectal cancer cells in vitro.



Figure 1. Chemical structure of Acetylshikonin. 


\subsection{Acetylshikonin Induced Apoptosis in HT29 Cells}

Flow cytometry was performed to detect the apoptosis manner with Annexin V and PI staining. Figure 3(a) and Figure 3(b) showed that the early apoptosis ratio increased from $15.4 \%$ to $42.6 \%$ with the treatment of Acetylshikonin (25, $50,100 \mu \mathrm{g} / \mathrm{ml}$ ) in HT29 cells in a dose-dependent manner. Thus, Acetylshikonin could induce apoptosis, especially early apoptosis in HT29 cells.

(a)

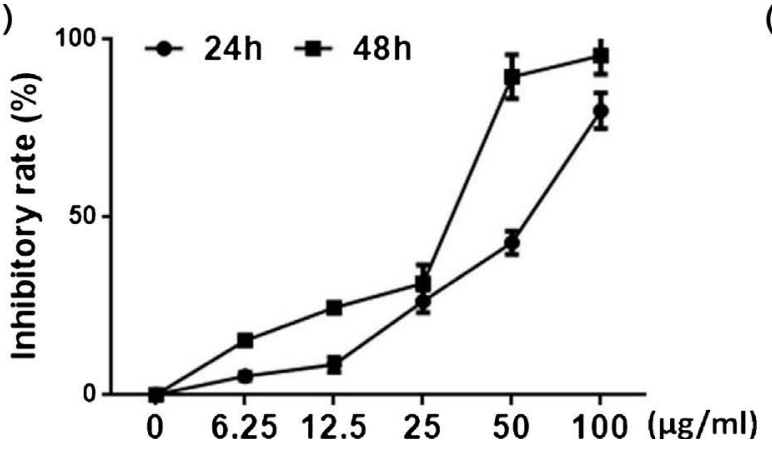

(b)

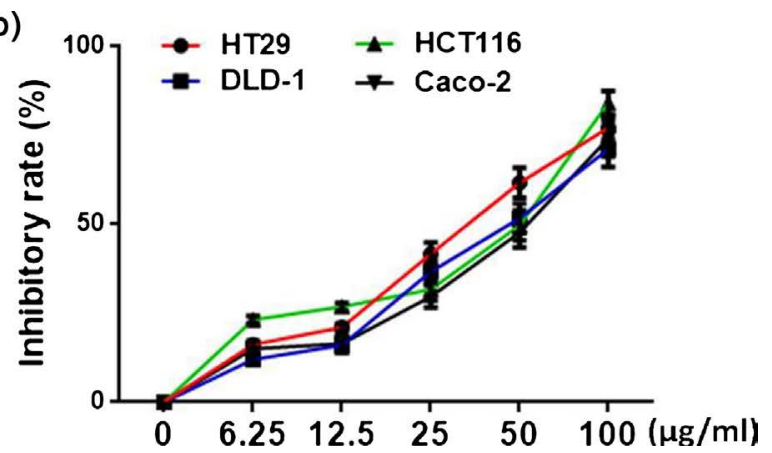

Figure 2. Effects of Acetylshikonin on colon carcinoma cells proliferation. (a): Cell inhibitory was measured using the MTT assay after treating cells with different concentrations $(6.25,12.5,25,50,100 \mu \mathrm{g} / \mathrm{ml}$ ) of Acetylshikonin for 24 and $48 \mathrm{~h}$. (b): HT29, HCT116, DLD-1 and Caco-2 cells were treated with various concentrations of Acetylshikonin for $48 \mathrm{~h}$. Cell inhibitory was analyzed by CCK- 8 assay. Results were obtained from three independent experiments, and the dots represent mean \pm SD.

(a)

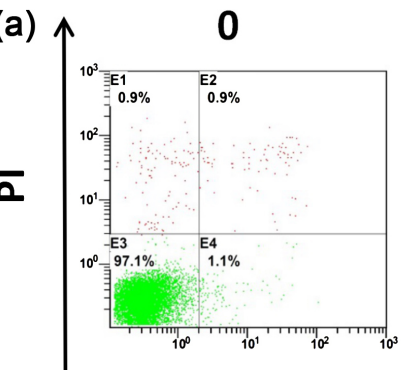

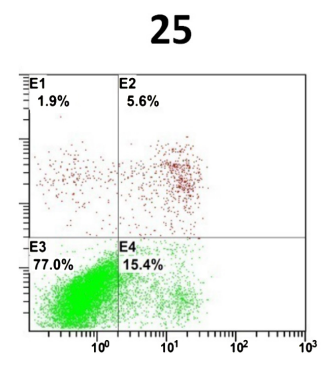

50



100

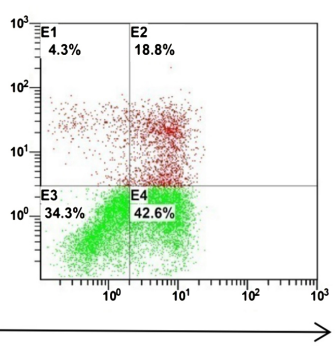

Concentration $(\mu \mathrm{g} / \mathrm{ml})$

Acetylshikonin

\section{Annexin V}

(b)

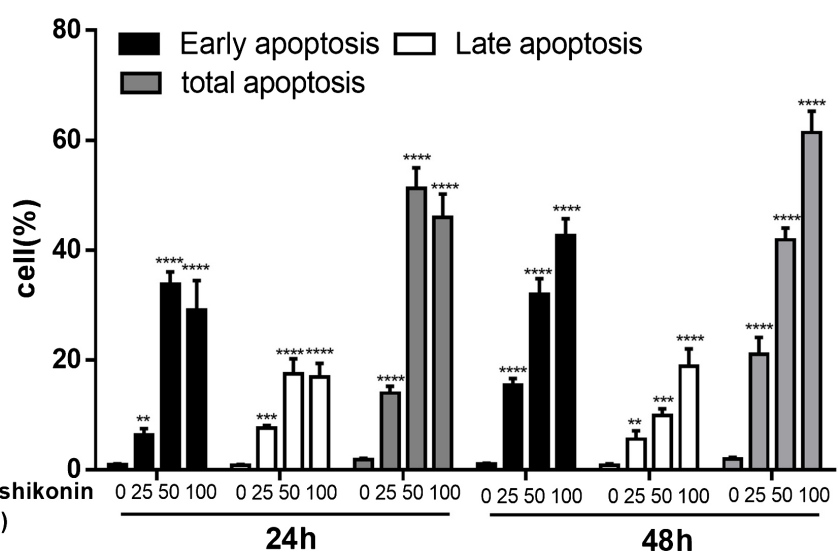

Figure 3. Acetylshikonin induced apoptosis in HT29 cells. (a): Apoptosis of HT29 cells was analyzed by flow cytometry. (b): Histograms demonstrate percentages of apoptotic cells in culture. Data are presented as means of three independent tests, and the dot represent mean $\pm \mathrm{SD} .{ }^{* * *} \mathrm{P}<0.00001,{ }^{* * *} \mathrm{P}<0.0001,{ }^{* *} \mathrm{P}<0.001,{ }^{*} \mathrm{P}<0.05$ 


\subsection{Acetylshikonin Induced Cell Cycle Arrested at G0/G1 in HT29 Cells}

The effect of Acetylshikonin on regulation of the cell cycle in HT29 cells was studied using Cycletest Plus DNA Reagent kit, and the results were evaluated by flow cytometry. As shown in Figure 4(a) and Figure 4(b), an increasing proportion of HT29 cells in G0/G1 phase was observed after treated with an increasing concentration of Acetylshikonin, while the percentage of HT29 cells in $S$ or $\mathrm{G} 2 / \mathrm{M}$ phase decreased.

\subsection{Activation of PI3K/Akt/mTOR Signaling Pathway Was Essen- tial for Acetylshikonin-Induced Apoptosis}

To further elucidate underlying mechanism of Acetylshikonin proapoptotic effect on HT29 cells, we evaluated whether PI3K/Akt/mTOR signaling pathway is involved in the apoptosis. Total protein from $0,25,50,100 \mu \mathrm{g} / \mathrm{ml}$ Acetylshikonin treated HT29 cells for $48 \mathrm{~h}$ were collected, and apoptotic proteins such as Akt, p-Akt, PI3K, p-PI3K, mTOR were determined by Western blot. With the increasing concentration of Acetylshikonin, the expression of these proteins including PI3K, p-PI3K, Akt, p-Akt, mTOR downregulated (Figure 5(a) and Figure 5(b)). Also, the results of RT-PCR showed that Acetylshikonin down-regulated the expression of these mRNAs including PI3K, Akt, mTOR in HT29 cells in a dose-dependent manner (Figure 5(c)).

To further determine the relationship between cell apoptosis and activation of
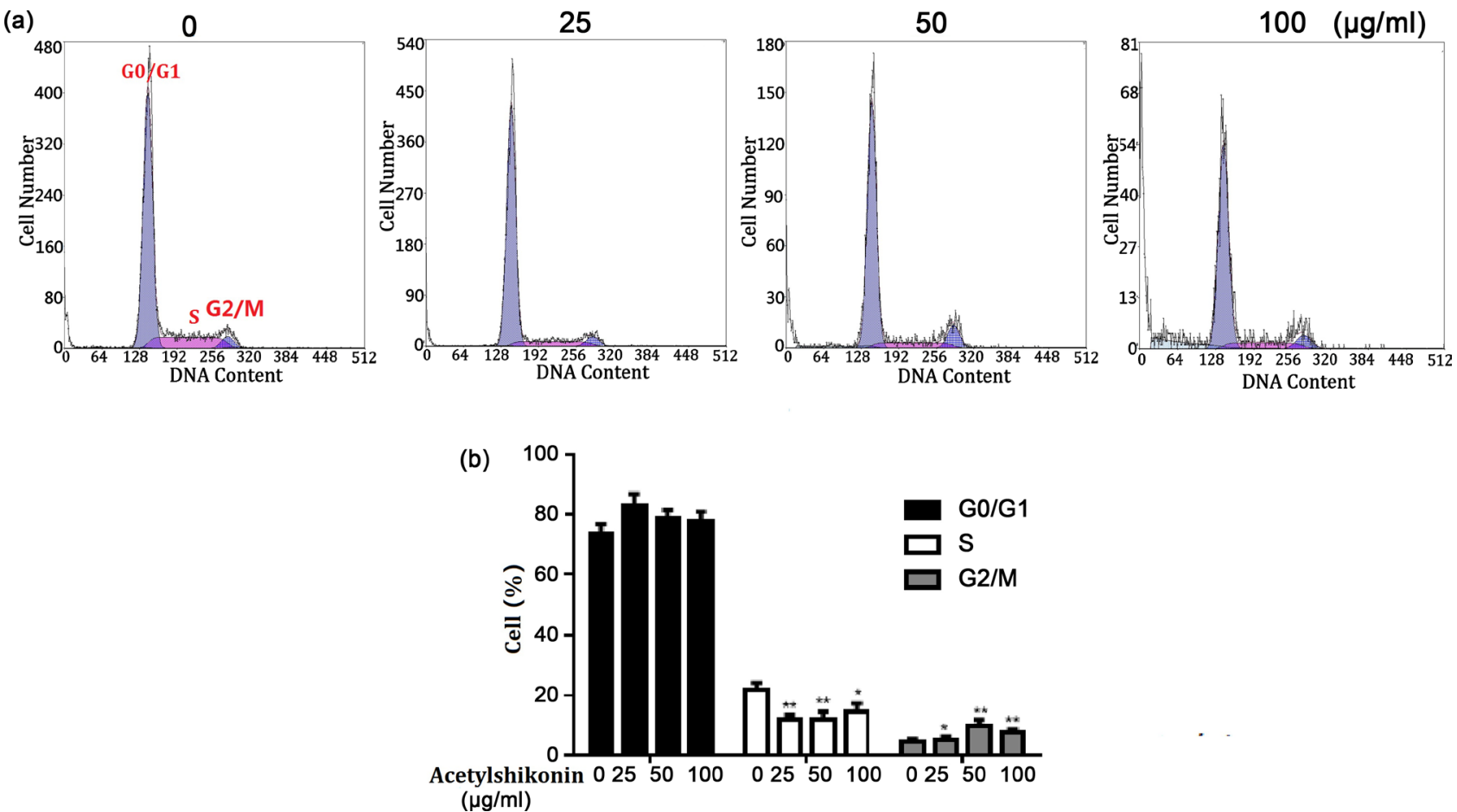

Figure 4. Acetylshikonin induced G0/G1 cell cycle arrest in HT29 cells. (a): Flow cytometric analysis of the proliferating HT29 cells. (b): Histograms demonstrate the percentage of tumor cells at different phases of the cell cycle. ${ }^{\star \star} \mathrm{P}<0.001$, ${ }^{\star} \mathrm{P}<0.05$ versus control (no Acetylshikonin) group. 
(a)

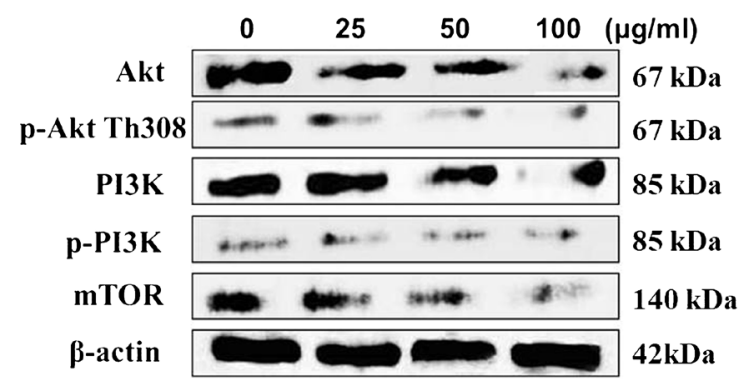

(b)

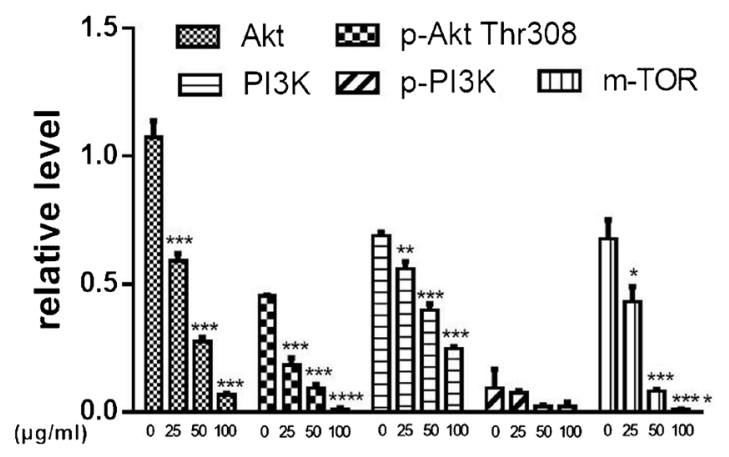

(c)

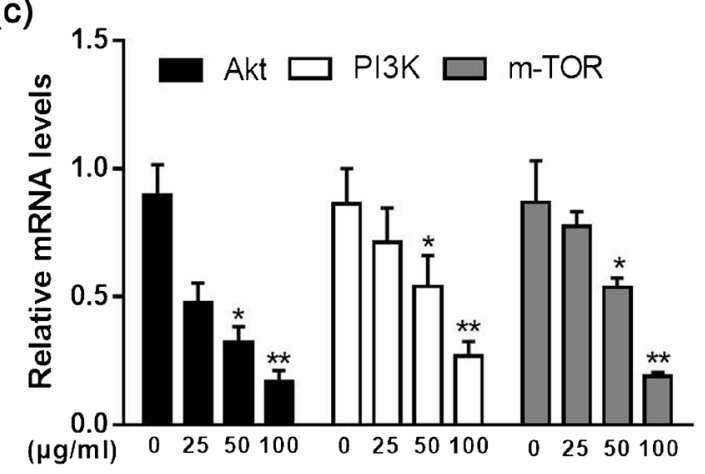

Figure 5. Effects of Acetylshikonin on Akt/PI3K/mTOR signalling pathway in HT29 cells. (a): Western blot analysed the protein expression of Akt, p-Akt, PI3K, p-PI3K, mTOR in HT29 cells when treated with or without Acetylshikonin. (b): Histograms demonstrate the relative expression level of proteins. (c): Akt, PI3K and mTOR gene expression were measured by real-time PCR treated with different concentrations of acetylshikonin for $48 \mathrm{~h} .{ }^{*} \mathrm{P}<$ $0.001,{ }^{\star} \mathrm{P}<0.05$ versus control (no Acetylshikonin) group.

PI3K/Akt/mTOR pathway, HT29 cells was exposed to Acetylshikonin and LY294002 (a PI3K/Akt inhibitor) for 48 h. HT29 cells treated with LY294002 plus $50 \mu \mathrm{g} / \mathrm{ml}$ Acetylshikonin had higher cell inhibitory rates and total apoptotic cells than those only treated with Acetylshikonin. But there was little change observed in the G0/G1 phase distribution (Figure 6(a) and Figure 6(b)). Similarly, Acetylshikonin plus LY294002 treatment caused a significant decrease in the ratio of p-Akt308/Akt, compared with that treated with Acetylshikonin alone (Figure 6(c) and Figure 6(d)).

These results demonstrated that apoptosis is induced by Acetylshikonin in HT29 cells via PI3K/Akt/mTOR signaling pathway.

\subsection{Acetylshikonin Inhibited the Growth of Xenografted Tumours in Nude Mice by PI3K/Akt/mTOR Signaling Pathway}

Based on the above results, we could thus conclude that Acetylshikonin is a promising anti-colorectal cancer agent. To further investigate the anti-tumour effect of Acetylshikonin, we established a subcutaneously transplanted colon carcinoma model using DLD-1 cells. After solid tumors were palpable (about $62.5 \mathrm{~m}^{3}$ ), mice were divided randomly into three groups and treated with intraperitoneal administration for a total of 13 days: control (1\% DMSO, every two 

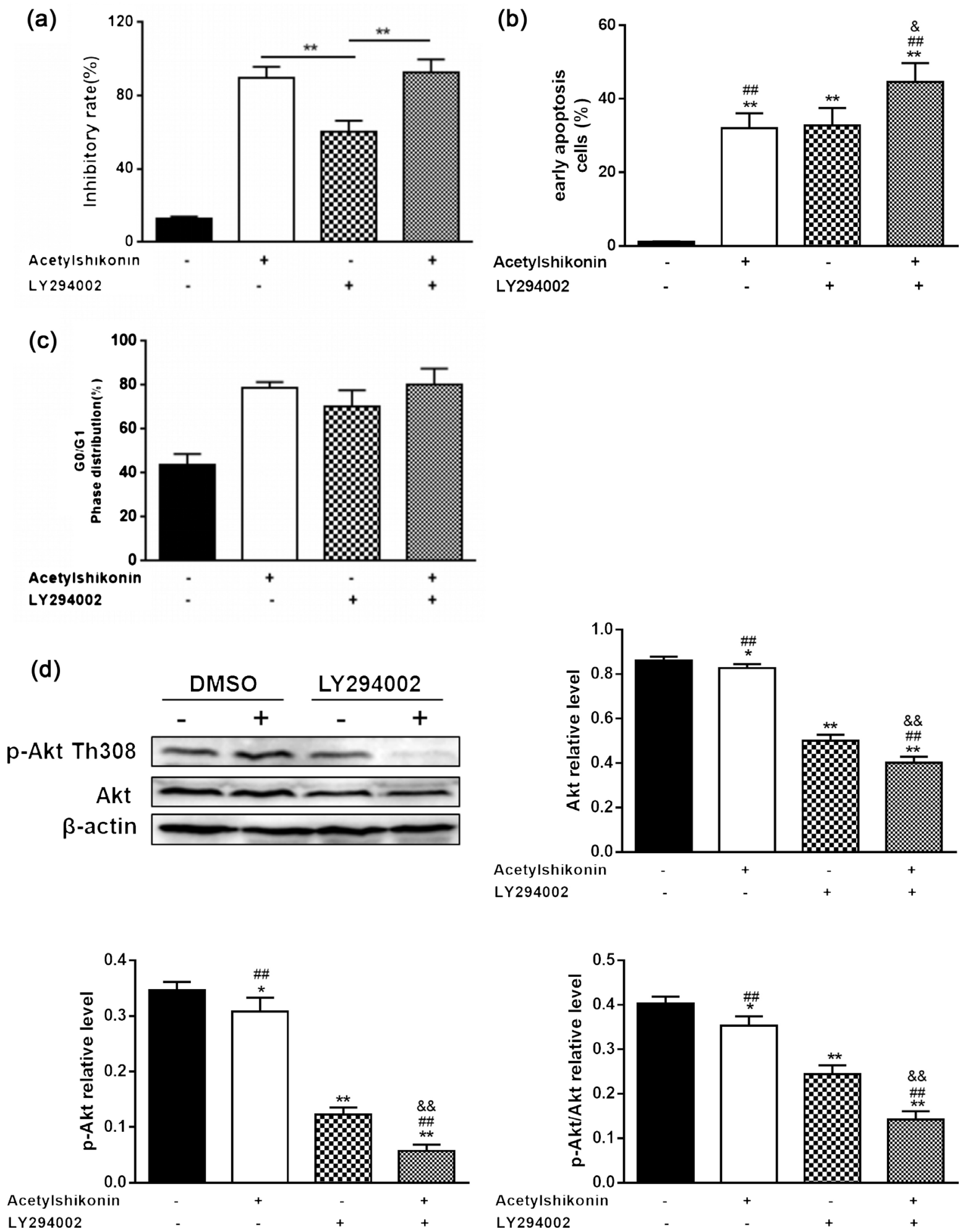

Figure 6. Effects of Acetylshikonin on inhibition of the PI3K/Akt/mTOR signaling pathway in HT29 cells. HT29 cells were pretreated with LY294002, then co-treated with $50 \mu \mathrm{g} / \mathrm{ml}$ Acetylshikonin for further $48 \mathrm{~h}$. (a): Cell viability was assessed by MTT assay. (b): Cell apoptosis was examined by Annexin V-FITC Apoptosis Detection Kit. (c): Cell cycle arrest was detected by flow cytometry. (d): Akt and p-Akt308 proteins were determined by Western blot. Data were presented as mean \pm SD of three independent tests. ${ }^{\star} \mathrm{P}<0.05,{ }^{* *} \mathrm{P}<0.001$ versus control (no Acetylshikonin and LY294002) group. ${ }^{*} \mathrm{P}<0.05,{ }^{\# \#} \mathrm{P}<0.001$ versus LY294002 group. ${ }^{\circledR} \mathrm{P}<0.05,{ }^{\& \&} \mathrm{P}<0.001$ versus Acetylshikonin group. 
days), Acetylshikonin (20 mg/kg in 1\% DMSO, every two days), irinotecan (66.7 $\mathrm{mg} / \mathrm{kg}$, every four days). No severe side effects were observed during the drug injected. The tumors were excised and weighed on day 13 when mice sacrificed. As shown in Figure 7(a) and Figure 7(b), both tumor weight and volume were significantly decreased in Acetylshikonin-treated group comparing with the control group. Furthermore, hematoxylin/eosin (H\&E) staining indicated that Acetylshikonin promoted necrosis and induced the disappearance of tumor cells. Compared to the control group, the expression of these proteins including PI3K, p-PI3K, Akt, p-Akt and mTOR in the tumour tissue were dramatically decreased in Acetylshikonin-treated group (Figure 7(c)). Thus, Acetylshikonin suppressed the growth of colorectal tumours by PI3K/Akt/mTOR signaling pathway in vivo.

Traditional Chinese medicine, envisioned as safer alternatives for their chemical counterparts, has been used for preventing cancer for centuries. Currently, effective components isolated from traditional Chinese medicines have become an important approach to discover anti-cancer drugs [13].

In our study, Acetylshikonin was extracted and isolated from the dried roots of $A$. euchroma. Acetylshikonin dramatically suppressed the proliferation of HT29 cells in the time and dose-dependent manner. The results of CCK-8 assay also revealed that Acetylshikonin at $25-100 \mu \mathrm{g} / \mathrm{ml}$ exhibited significant inhibition of cell proliferation in human colorectal cancer cells including HT29, DLD-1, HCT116 and Caco-2. These findings indicated that Acetylshikonin has the potential to develop into a novel anti-tumor drug for colorectal cancer.

Apoptosis is a genetically programmed process leading to cell death, which primarily functions to eliminate senescent or altered cells that are useless or harmful to health. In contrast, abnormal changes in apoptotic mechanisms that promote deficient programmed cell death are closely related to the occurrence and development of tumours [14]. Recent evidence has indicated that selective induction of apoptosis in tumour cells is the most direct and efficacy method to treat tumours [15]. In our study, to further clarify the inhibitory effects of Acetylshikonin on colorectal cancer, Annexin V/PI staining was applied to detect the effect of Acetylshikonin on HT29 cells apoptosis. The proportion of total apoptotic cells (particularly early apoptosis) induced by Acetylshikonin was significantly increased dose-dependently.

Cell cycle progression plays an important role in tumour growth, and its regulation is an effective strategy to control tumour growth. It has been reported that the active ingredients of traditional Chinese medicine can arrest tumour cells at different cell cycle, which in turn inhibit tumour cell growth, proliferation, and induce apoptosis [16] [17]. In present study, Acetylshikonin blocked cell cycle progression at G0/G1 phase, which demonstrated that Acetylshikonin interfered with the normal tumour cell cycle and inhibited tumour growth.

$\mathrm{PI} 3 \mathrm{~K} / \mathrm{Akt} / \mathrm{mTOR}$ pathway regulates multiple cellular processes such as growth [18], proliferation [19], cell cycle progression [20], motility [18], adhesion [21], 



Control



Acetylshikonin

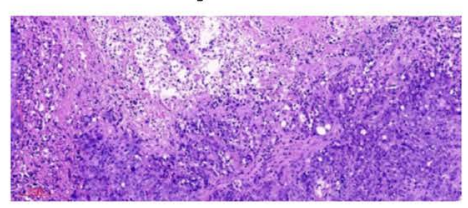

Irinotecan

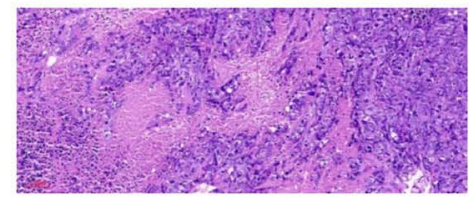

(c) Control Acetylshikonin Irinotecan
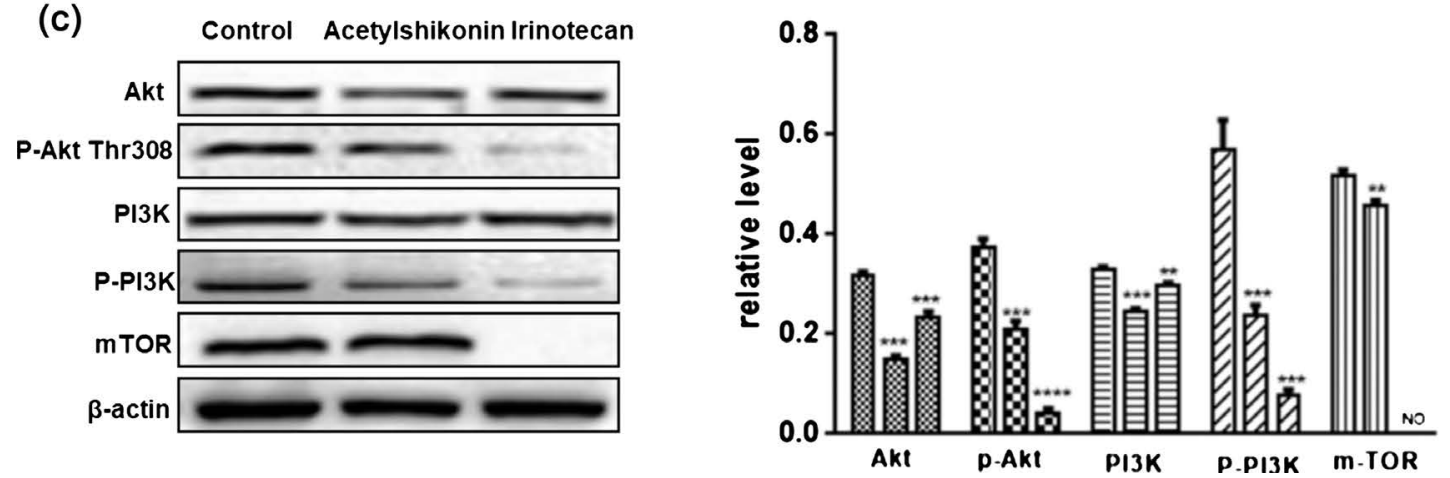

Figure 7. Acetylshikonin inhibited the growth of xenografted tumours in nude mice by PI3K/Akt/mTOR signaling pathway. Male NOD/SCID mice were injected with DLD-1 cells into the subcutaneous tissue of the back region. Xenograft model was established successfully when tumour average size of $62.5 \mathrm{~mm}^{3}$, and the different drugs was intraperitoneally injected for 13 days: Control group (1\% DMSO, every two days), Acetylshikonin-treated group (20 mg/kg in 1\% DMSO, every two days), irinotecan positive group (66.7 mg/kg, every four days). (a): Body weights and tumour volumes were measured once a day. (b): Images, tumour weights and H\&E staining were measured after drugs treated for 13 days. (c): The expression of these proteins including PI3K, p-PI3K, Akt, p-Akt and mTOR in the tumour tissue of control, Acetylshikonin and irinotecan treatment groups were analysed by Western blot. Data are presented as mean $\pm \mathrm{SD}$. ${ }^{\star} \mathrm{P}<0.05,{ }^{* *} \mathrm{P}<0.001,{ }^{* *} \mathrm{P}<0.0001,{ }^{* * * *} \mathrm{P}<0.00001$ compared to the control group. 
and angiogenesis [22]. Previous studies have indicated that abnormal activation of PI3K/Akt/mTOR signaling pathway is commonly involved in the development and progression of various tumours [23] [24] [25], especially colorectal cancer [26]. Akt is one of core target proteins to downstream of PI3K, which relies on the phosphorylation at the Thr308 site of the catalytic domain and the Ser473 site of the regulatory domain of the C-terminus. In this study, we found Acetylshikonin down-regulated Akt phosphorylation at Thr308. However, the precise molecular mechanism by which Acetylshikonin deactivates Akt should be further investigated. Combining Acetylshikonin with LY294002 largely increased the rate of cell inhibitory, early apoptosis and G0/G1 phase distribution of HT29. As a targeted anti-cancer drug LY294002, has also been reported in several cancers, Acetylshikonin may also be useful for improving anti-cancer activity of PI3K/Akt/mTOR-targeting drugs.

Further experiments were conducted in vivo based on the preliminary in vitro research. Thus, we established the transplanted colon carcinoma model of human DLD-1 cells in NOD/SCID mice to observe the anti-tumor effect of Acetylshikonin in vivo. Acetylshikonin obviously inhibited the growth of colon tumours with no body weight changes. Additionally, there was little difference in weight and pathology of tumours between the Acetylshikonin-treated group and irinotecan positive group. Furthermore, Acetylshikonin could inhibit PI3K/Akt/mTOR pathway in vivo by Western blot analysis, which is in ageement with the results in vitro.

\section{Conclusions}

In conclusion, our study demonstrated that Acetylshikonin possessed the anti-cancer activity in human colorectal cancer. Acetylshikonin effectively inhibited cell proliferation, induced cell cycle arrested at G0/G1 and promoted colorectal cancer cells apoptosis in vitro. Colorectal cancer growth was also suppressed by Acetylshikonin in vivo. Meanwhile, activation of PI3K/Akt/mTOR signaling pathway played a crucial role in the treatment with Acetylshikonin. Collectively, these findings reveal that Acetylshikonin may serve as an anti-tumour candidate for colorectal cancer treatment.

\section{Authors' Contributions}

$\mathrm{XBZ}$ and YZZ developed the concept of this study. YZZ, XBZ, YZ, YZ, YYL, QLH, ZH, YCW and HY designed and performed experiments and analysed data. YZZ, XBZ and XBZ prepared the draft and final version of the manuscript. All authors read and approved the final manuscript.

\section{Acknowledgements}

Financial support was provided by National Natural Science Foundation of China (81573932, 81603592), Medical Research Foundation of Guangdong Province (A2017414, B2017044), Natural Science Foundation of Guangdong 
Province (2016A030310356, 2014A030307001), the Science and Technology Fund of Zhanjiang (2014A01014), Research Foundation of Guangdong Medical University (M2016021, M2013003) and Traditional Chinese Medicine Research Foundation of Guangdong Provincial Bureau (20151260).

\section{Conflicts of Interest}

The authors declare that they have no conflict of interest.

\section{Availability of Data and Materials}

The datasets used and/or analyzed during the current study are available from the corresponding author on reasonable request.

\section{Consent for Publication}

All of authors consent to publication of this study in Journal of Chinese Medicine.

\section{Ethics Approval and Consent to Participate}

Animal protocol was conducted according to the animal procedure approved by Animal Ethics Committee, Guangdong Key Laboratory for Research and Development of Natural Drugs, Guangdong Medical University (SYXK (GUANGDONG) 2016-0013).

\section{References}

[1] Benard, F., Barkun, A.N., Martel, M. and von Renteln, D. (2018) Systematic Review of Colorectal Cancer Screening Guidelines for Average-Risk Adults: Summarizing the Current Global Recommendations. World Journal of Gastroenterology, 24, 124-138. https://doi.org/10.3748/wjg.v24.i1.124

[2] Miguel, A., Azevedo, L.F., Araujo, M. and Pereira, A.C. (2012) Frequency of Adverse Drug Reactions in Hospitalized Patients: A Systematic Review and Meta-Analysis. Pharmacoepidemiology and Drug Safety, 21, 1139-1154. https://doi.org/10.1002/pds.3309

[3] Liang, W., Cai, A., Chen, G., Xi, H., Wu, X., Cui, J., Zhang, K., Zhao, X., Yu, J., Wei, B. and Chen, L. (2016) Shikonin Induces Mitochondria-Mediated Apoptosis and Enhances Chemotherapeutic Sensitivity of Gastric Cancer through Reactive Oxygen Species. Scientific Reports, 6, Article ID: 38267. https://doi.org/10.1038/srep38267

[4] Li, X., Fan, X.X., Jiang, Z.B., Loo, W.T., Yao, X.J., Leung, E.L., Chow, L.W. and Liu, L. (2017) Shikonin Inhibits Gefitinib-Resistant Non-Small Cell Lung Cancer by Inhibiting TrxR and Activating the EGFR Proteasomal Degradation Pathway. Pharmacological Research, 115, 45-55. https://doi.org/10.1016/j.phrs.2016.11.011

[5] Wang, Y., Zhou, Y., Jia, G., Han, B., Liu, J., Teng, Y., Lv, J., Song, Z., Li, Y., Ji, L., Pan, S., Jiang, H. and Sun, B. (2014) Shikonin Suppresses Tumour Growth and Synergizes with Gemcitabine in a Pancreatic Cancer Xenograft Model: Involvement of NF- $\kappa$ B Signalling Pathway. Biochemical Pharmacology, 88, 322-333. https://doi.org/10.1016/j.bcp.2014.01.041

[6] Tang, S., Chen, A., Zhou, X., Zeng, L., Liu, M. and Wang, X. (2017) Assessment of 
the Inhibition Risk of Shikonin on Cytochrome P450 via Cocktail Inhibition Assay. Toxicology Letters, 281, 74-83. https://doi.org/10.1016/j.toxlet.2017.09.014

[7] Huang, G., Zhao, H.R., Meng, Q.Q., Zhang, Q.J., Dong, J.Y., Zhu, B.Q. and Li, S.S. (2018) Synthesis and Biological Evaluation of Sulfur-Containing Shikonin Oxime Derivatives as Potential Antineoplastic Agents. European Journal of Medicinal Chemistry, 143, 166-181. https://doi.org/10.1016/j.ejmech.2017.11.031

[8] Ning, X., Qi, H., Li, R., Li, Y., Jin, Y., McNutt, M.A., Liu, J. and Yin, Y. (2017) Discovery of Novel Naphthoquinone Derivatives as Inhibitors of the Tumour Cell Specific M2 Isoform of Pyruvate Kinase. European Journal of Medicinal Chemistry, 138, 343-352. https://doi.org/10.1016/j.ejmech.2017.06.064

[9] Park, S.H., Phuc, N.M., Lee, J., Wu, Z., Kim, J., Kim, H., Kim, N.D., Lee, T., Song, K.S. and Liu, K.H. (2017) Identification of Acetylshikonin as the Novel CYP2J2 Inhibitor with Anti-Cancer Activity in HepG2 Cells. Phytomedicine, 24, 134-140. https://doi.org/10.1016/j.phymed.2016.12.001

[10] Cho, S.C. and Choi, B.Y. (2015) Acetylshikonin Inhibits Human Pancreatic PANC-1 Cancer Cell Proliferation by Suppressing the NF- $\kappa$ B Activity. Biomolecules \& Therapeutics, 23, 428-433. https://doi.org/10.4062/biomolther.2015.102

[11] Zou, Y., Lin, J.T., Li, W.Y., Wu, Z.G., He, Z.W., Huang, G.L., Wang, J., Ye, C.G., Cheng, X.Y., Ding, C.C., Zheng, X.B. and Chi, H.G. (2016) Huangqin-Tang Ameliorates Dextran Sodium Sulphate-Induced Colitis by Regulating Intestinal Epithelial Cell Homeostasis, Inflammation and Immune Response. Scientific Reports, 6, Article ID: 39299. https://doi.org/10.1038/srep39299

[12] Ko, F.N., Lee, Y.S., Kuo, S.C., Chang, Y.S. and Teng, C.M. (1995) Inhibition on Platelet Activation by Shikonin Derivatives Isolated from Arnebia euchroma. Biochimica et Biophysica Acta (BBA), Molecular Cell Research, 1268, 329-334. https://doi.org/10.1016/0167-4889(95)00078-7

[13] Qi, F., Zhao, L., Zhou, A., Zhang, B., Li, A., Wang, Z. and Han, J. (2015) The Advantages of Using Traditional Chinese Medicine as an Adjunctive Therapy in the Whole Course of Cancer Treatment instead of Only Terminal Stage of Cancer. BioScience Trends, 9, 16-34. https://doi.org/10.5582/bst.2015.01019

[14] Marquez-Jurado, S., Diaz-Colunga, J., Das Neves, R.P., Martinez-Lorente, A., Almazan, F., Guantes, R. and Iborra, F.J. (2018) Mitochondrial Levels Determine Variability in Cell Death by Modulating Apoptotic Gene Expression. Nature Communications, 9, 389. https://doi.org/10.1038/s41467-017-02787-4

[15] Fulda, S. (2018) Cell Death-Based Treatment of Glioblastoma. Cell Death \& Disease, 9, 121. https://doi.org/10.1038/s41419-017-0021-8

[16] Zheng, T., Que, Z., Jiao, L., Kang, Y., Gong, Y., Yao, J., Ma, C., Bi, L., Dong, Q., Zhao, X. and Xu, L. (2017) Herbal Formula YYJD Inhibits Tumour Growth by Inducing Cell Cycle Arrest and Senescence in Lung Cancer. Scientific Reports, 7, Article No. 4984. https://doi.org/10.1038/s41598-017-05146-X

[17] Chen, X., Wu, Q.S., Meng, F.C., Tang, Z.H., Chen, X., Lin, L.G., Chen, P., Qiang, W.A., Wang, Y.T., Zhang, Q.W. and Lu, J.J. (2016) Chikusetsusaponin IVa Methyl Ester Induces G1 Cell Cycle Arrest, Triggers Apoptosis and Inhibits Migration and Invasion in Ovarian Cancer Cells. Phytomedicine, 23, 1555-1565.

https://doi.org/10.1016/j.phymed.2016.09.002

[18] Chen, D., Lin, X., Zhang, C., Liu, Z., Chen, Z., Li, Z., Wang, J., Li, B., Hu, Y., Dong, B., Shen, L., Ji, J., Gao, J. and Zhang, X. (2018) Dual PI3K/mTOR Inhibitor BEZ235 as a Promising Therapeutic Strategy against Paclitaxel-Resistant Gastric Cancer via Targeting PI3K/Akt/mTOR Pathway. Cell Death \& Disease, 9, 123. 
https://doi.org/10.1038/s41419-017-0132-2

[19] Pan, Y., Wang, N., Xia, P., Wang, E., Guo, Q. and Ye, Z. (2018) Inhibition of Rac1 Ameliorates Neuronal Oxidative Stress Damage via Reducing Bcl-2/Racl Complex Formation in Mitochondria through PI3K/Akt/mTOR Pathway. Experimental Neurology, 300, 149-166. https://doi.org/10.1016/j.expneurol.2017.10.030

[20] Wang, F., Wang, Q., Zhou, Z.W., Yu, S.N., Pan, S.T., He, Z.X., Zhang, X., Wang, D., Yang, Y.X., Yang, T., Sun, T., Li, M., Qiu, J.X. and Zhou, S.F. (2015) Plumbagin Induces Cell Cycle Arrest and Autophagy and Suppresses Epithelial to Mesenchymal Transition Involving PI3K/Akt/mTOR-Mediated Pathway in Human Pancreatic Cancer Cells. Drug Design Development and Therapy, 9, 537-560.

[21] Ackermann, M.A., King, B., Lieberman, N.A.P., Bobbili, P.J., Rudloff, M., Berndsen, C.E., Wright, N.T. and Hecker, P.A. (2017) Kontrogianni-Konstantopoulos A. Novel Obscurins Mediate Cardiomyocyte Adhesion and Size via the PI3K/AKT/mTOR Signalling Pathway. Journal of Molecular and Cellular Cardiology, 111, 27-39. https://doi.org/10.1016/j.yjmcc.2017.08.004

[22] Xie, Y., Naizabekov, S., Chen, Z. and Tokay, T. (2016) Power of PTEN/AKT: Molecular Switch between Tumour Suppressors and Oncogenes. Oncology Letters, 12, 375-378. https://doi.org/10.3892/ol.2016.4636

[23] Courtney, K.D., Corcoran, R.B. and Engelman, J.A. (2010) The PI3K Pathway as Drug Target in Human Cancer. Journal of Clinical Oncology, 28, 1075-1083. https://doi.org/10.1200/JCO.2009.25.3641

[24] Tonlaar, N., Galoforo, S., Thibodeau, B.J., Ahmed, S., Wilson, T.G., Yumpo Cardenas, P., Marples, B. and Wilson, G.D. (2017) Antitumour Activity of the Dual PI3K/MTOR Inhibitor, PF-04691502, in Combination with Radiation in Head and Neck Cancer. Radiotherapy and Oncology, 124, 504-512. https://doi.org/10.1016/j.radonc.2017.08.001

[25] Pandurangan, A.K. (1970) Potential Targets for Prevention of Colorectal Cancer: A Focus on PI3K/Akt/mTOR and Wnt Pathways. Asian Pacific Journal of Cancer Prevention, 14, 2201-2205. https://doi.org/10.7314/APJCP.2013.14.4.2201

[26] Liu, Y., Bi, T., Wang, Z., Wu, G., Qian, L., Gao, Q. and Shen, G. (2016) Oxymatrine Synergistically Enhances Antitumour Activity of Oxaliplatin in Colon Carcinoma through PI3K/AKT/mTOR Pathway. Apoptosis, 21, 1398-1407.

https://doi.org/10.1007/s10495-016-1297-3 


\section{Abbreviations}

$\mathrm{CHCl}_{3}$, Chloroform

MTT, Methyl Thiazolyl Tetrazolium

CCK-8, Cell Counting Kit-8

RT-PCR, Reverse Transcription Polymerase Chain Reaction

CRC, Colorectal Cancer

NMR, Nuclear Magnetic Resonance

TMS, Tetramethylsilane

ODS, Octadecylsilane

ATCC, American Type Culture Collection

qRT-PCR, Quantitative Reverse Transcription Polymerase Chain Reaction DMSO, Dimethyl Sulphoxide

PI, Propidium lodide

H\&E, Hematoxylin/eosin

PI3K, Phosphatidyl Inositol (-3) Kinase

p-PI3K, Phosphorylated Phosphatidyl Inositol (-3) Kinase

Akt, Protein Kinase B

p-Akt, Phosphorylated Protein Kinase B

mTOR, Mammalian Target of Rapamycin 